\title{
Caracterização da anatomia foliar de cafeeiros arábica em diferentes períodos sazonais
}

\author{
Rachel Benetti Queiroz-Voltan ${ }^{1 *}$ \\ Carla Fernanda Nardin ${ }^{1}$ \\ Luiz Carlos Fazuoli ${ }^{2}$ \\ Masako Toma Braghini ${ }^{2}$
Instituto Agronômico, Centro de Pesquisa e Desenvolvimento de Recursos Genéticos Vegetais Avenida Barão de Itapura, 1481, Caixa Postal 28, CEP 13020-902, Campinas - SP, Brasil
${ }^{2}$ Instituto Agronômico, Centro de Análise e Pesquisa Tecnológica do Agronegócio do Café
* Autor para correspondência
rachelqv@yahoo.com.br

Submetido em 06/03/2014

Aceito para publicação em 18/08/2014

\section{Resumo}

A seca é um dos fatores que limitam o desenvolvimento e a produtividade do cafeeiro. O objetivo deste estudo foi analisar a anatomia de folhas desenvolvidas nos períodos chuvoso e seco, em nove cafeeiros de Coffea arabica L., descritos anteriormente como tolerantes ou sensíveis a déficit hídrico, para identificar caracteres estruturais de tolerância à seca. Foram mensurados o tamanho e a densidade de estômatos e células epidérmicas, a espessura das epidermes e do mesofilo, o diâmetro do pecíolo e da nervura principal, a espessura do floema e xilema na nervura principal e no pecíolo e o diâmetro e a frequência de elementos de vasos do xilema. Diferenças observadas entre a estrutura foliar dos cafeeiros avaliados e as condições de desenvolvimento da folha (períodos chuvoso e seco) indicaram que há uma plasticidade anatômica favorável em relação às condições de seca. Espessuras maiores do parênquima paliçádico e do limbo total e raios maiores do floema e xilema no pecíolo e na nervura principal foram considerados características estruturais favoráveis para suportar a escassez de água e estas poderiam ser utilizadas como critérios para selecionar cultivares tolerantes à seca. Foram considerados mais adaptados à seca os seguintes cafeeiros: Geisha, Semperflorens, BA10, IAC H 8105-7, IAC H 8421-2 e a cultivar Catuaí Vermelho IAC 81.

Palavras-chave: Adaptações estruturais; Coffea arabica; Mesofilo; Tolerância à seca

\section{Abstract}

Leaf anatomy characterization of Coffea arabica plants at different seasonal periods. Drought is among the hindering factors for coffee plant growth and yield. This study aimed to analyze the anatomy of leaves grown during the rainy and dry seasons in nine Coffea arabica L. plants, previously described as tolerant or sensitive to water deficit, in order to identify structural features of drought tolerance. We measured the size and density of stomata and epidermal cells, the thickness of epidermis and mesophyll, the diameter of petiole and midrib, the thickness of phloem and xylem in the midrib and petiole, and the diameter and frequency of elements of xylem vessels. Differences observed between the leaf structure of coffee plants evaluated and the leaf growth 
conditions (rainy and dry seasons) indicated that there is a favorable anatomical plasticity regarding drought conditions. Thicker palisade parenchyma and total limbo, larger radii of phloem and xylem in the petiole and midrib were considered as favorable structural features to cope with water scarcity and they could be used as criteria to select drought-resistant cultivars. The following coffee plants were considered as more adapted to drought: Geisha, Semperflorens, BA 10, IAC H 8105-7, IAC H 8421-2, and the cultivar Catuaí Vermelho IAC 81.

Key words: Coffea arabica; Drought tolerance; Mesophyll; Structural adaptations

\section{Introdução}

A deficiência hídrica no solo é um dos principais fatores ambientais que limitam o desenvolvimento das plantas e sua produtividade. O cafeeiro é muito afetado pelo déficit hídrico (ARRUDA; GRANDE, 2003; GOMES et al., 2007). Diminuições na oferta de água podem reduzir o crescimento do cafeeiro, mesmo não sendo visíveis os caracteres externos de deficiência hídrica (DAMATTA, 2004). Portanto, o conhecimento dos mecanismos que as plantas utilizam para se manterem túrgidas em condições de déficit de água é importante para a obtenção de cultivares tolerantes à seca (BATISTA et al., 2010).

Estudos vêm sendo realizados com plantas do gênero Coffea, visando identificar materiais genéticos resistentes ou tolerantes à seca, como o de Mazzafera e Carvalho (1987). Estes autores avaliaram diversos cafeeiros do Banco Ativo de Germoplasma do Instituto Agronômico (BAG IAC), híbridos F1 e os seus genitores, entre materiais procedentes da Índia e da África, com as cultivares Mundo Novo e Catuaí Vermelho de Coffea arabica que, após um longo período de estiagem, apresentaram características de tolerância hídrica. As plantas BA 10 (Índia), IAC 1521-2 (Costa Rica) e IAC 1120-16 (Tanzânia) foram indicadas como as mais promissoras, após avaliação de dados de treze anos de produção e de quatro anos (não consecutivos) de observações visuais de campo, em relação à tolerância à seca. O cafeeiro BA10 apresentou também, em termos de produção, boas combinações com as cultivares Mundo Novo e Catuaí.

Outros estudos realizados, também em materiais genéticos do Banco de Germoplasma do IAC, por Almeida et al. (2007), sugeriram que alguns cafeeiros sobreviveram ao estresse hídrico a que foram submetidos no experimento de vasos, provavelmente devido à estratégia de murchamento das folhas e de ajustamento osmótico via prolina. Os diferentes cafeeiros foram classificados em: Tolerantes (Semperflorens, EP 131 C188B, EP 131 C91, Laurina, Moca Grande); Intermediários (Catuaí Vermelho IAC 81, Geisha, BA 10, EP 131 C188A, EP 473 C612, EP 473 C879, Caturra Amarelo, Caturra Vermelho, Villa Sarchí, Barbuk Sudan, Ibaaré, Agaro, C. dewevrei var. abeokutae e Mundo Novo) e Sensíveis (Apoatã e Robustão Capixaba de C. canephora e Bourbon Vermelho de C. arabica). As cultivares Siriema (GRISI et al., 2008), Bourbon Amarelo IAC 06 e Catimor UFV 5390 (BATISTA et al., 2010) foram consideradas pelos autores com características anatômicas e fisiológicas potenciais de tolerância ao déficit hídrico.

A anatomia foliar varia em função da radiação solar, temperatura, quantidade de água disponível no ambiente e nutrientes do solo, conferindo assim características adaptativas para que a planta tenha um ótimo desenvolvimento. Portanto, as folhas variam em área, espessura, forma, concentração de nutrientes e capacidade de troca gasosa, que necessariamente precisam ser ajustadas à economia de carbono, incluindo massa seca foliar por área, longevidade, concentração de nitrogênio por massa e taxa fotossintética líquida máxima, para resultar em folhas com alta condutância hidráulica. Folhas que apresentam alta densidade de nervuras e tecidos xilemáticos desenvolvidos nas nervuras possuem alta condutância hidráulica, que por sua vez está relacionada com a maior espessura do parênquima paliçádico, com a razão entre os parênquimas paliçádico e o esponjoso do mesofilo foliar e, com a espessura foliar (SACK; HOLBROOK, 2006; BRODRIBB et al., 2007).

A estrutura foliar geralmente é um bom indicador da disponibilidade de água e luz presente no habitat de uma espécie e, por ser um órgão com maior plasticidade 
na sua resposta às condições ambientes, sua estrutura responde mais do que o caule e a raiz aos efeitos do estresse ambiental (MARCHI et al., 2008). Genótipos que ocorrem em ambientes secos e ensolarados geralmente possuem folhas mais espessas, maiores massas específicas e densidade estomática, menores dimensões das células do mesofilo e com poucos espaços intercelulares, cutícula espessa, dentre outros, quando comparados com os desenvolvidos em ambientes úmidos e sombreados (MEDIAVILLA et al., 2001).

Este trabalho objetivou estudar a variação da anatomia foliar, de nove cafeeiros de Coffea arabica L., após períodos chuvoso e seco, visando identificar estruturas adaptativas de tolerância à seca em cafeeiros indicados como promissores para essa condição.

\section{Material e Métodos}

As avaliações foram realizadas em nove cafeeiros (Coffea arabica L.), pertencentes ao BAG IAC, sendo seis da coleção e três do experimento de progênie EP 131, instalados no Centro Experimental de Campinas/ IAC-SP (latitude $22^{\circ} 54$ 'S , longitude $47^{\circ} 05^{\prime}$ ' W e $674 \mathrm{~m}$ de altitude). As idades das plantas eram iguais dentro da coleção e da progênie e, diferentes entre si. Os cafeeiros avaliados foram:

1 - Semperflorens-Coleção 6 (C. arabica var. semperflorens), 2 - BA10-Coleção 2 (Progênie de $C$. arabica com resistência à ferrugem selecionada em Mysore, Índia), 3 - Nacional-Coleção 9 (C. arabica 'Nacional'), 4 - Gláucia-Coleção 7 (C. arabica var. glaucia), 5 - Geisha-Coleção 1 (C. arabica var. geisha, introduzida da Etiópia), 6 - 'Catuaí Vermelho IAC 81' - Coleção 4; 7 - IAC H 8105-7 ('Catuaí Vermelho IAC 81' X BA10) EP 131 planta 91, 8 - IAC H 84212 ('Mundo Novo IAC 471-5' X BA10) EP 131 planta 188, 9 - 'Catuaí Vermelho IAC 81' EP 131 planta 31. O cafeeiro 'Catuaí Vermelho IAC 81' é uma cultivar de $C$. arabica de porte baixo, amplamente plantada no Brasil.

A escolha por esses cafeeiros foi baseada nos resultados obtidos por Mazzafera e Carvalho (1987) e Almeida et al. (2007), observações efetuadas por pesquisadores do Centro de Café em plantas do BAG
IAC, que apresentaram características de tolerância à seca. Além disso, também foi incluída nas análises do presente trabalho a cultivar Nacional, que apresentou sensibilidade em observações de campo.

Foram coletadas cinco folhas totalmente expandidas do terço superior de cada planta, de ramos plagiotrópicos, localizados na face voltada para o norte. As folhas foram retiradas do $3^{\circ}$ nó do ramo, a partir do ápice, em dois períodos do ano de 2011, chuvoso (abril) e seco (setembro). Segmentos de $0,25 \mathrm{~cm}^{2}$ foram retirados da região mediana do limbo e da região da nervura principal e também, segmentos de $0,5 \mathrm{~cm}$ do pecíolo. Estas amostras foram fixadas em solução de formaldeído-ácido acético-álcool etílico $50 \%$ e submetidas a vácuo por $48 \mathrm{~h}$. Posteriormente, as amostras foram desidratadas em série alcoólica-etílica, incluídas em parafina e seccionadas transversalmente em micrótomo rotativo manual com 10 $\mu \mathrm{m}$ de espessura (JOHANSEN, 1940). Dos segmentos do limbo, também foram preparados blocos para serem realizados cortes paradérmicos, nas regiões da epiderme nas faces abaxial e adaxial, e do parênquima esponjoso e paliçádico. As lâminas foram coradas com safraninaazul de alcian, montadas em adesivo "Permount" e analisadas com o auxílio de um microscópio óptico Leica modelo DMLB, acoplado a uma câmera digital MOTICAM 2300.

As temperaturas e precipitações médias mensais dos locais de experimentação, no período de setembro de 2010 a outubro de 2011, foram obtidas no Centro Integrado de Informações Agrometeorológicas CIIAGRO (2013) (Figura 1).

Estimou-se a densidade de estômatos e das células epidérmicas na face abaxial, para se obter o índice estomático (IE). O IE, segundo Cutter (1986), tem sido muito utilizado para comparar diferentes materiais genéticos, sejam eles cultivares ou espécies de igual ou de diferentes ploidias. É um valor razoavelmente constante, sendo afetado em grande parte somente pela umidade. Os diâmetros, polar e equatorial, dos estômatos também foram mensurados.

As espessuras das faces da epiderme (abaxial e adaxial), dos parênquimas (paliçádico e esponjoso) foram mensuradas e, do somatório destas, obteve-se a 
FIGURA 1: Médias mensais de temperatura e precipitação pluviométrica, referentes ao período de setembro de 2010 a outubro de 2011 , no Centro Experimental do Instituto Agronômico, Campinas, SP. Fonte: CIIAGRO (2013).

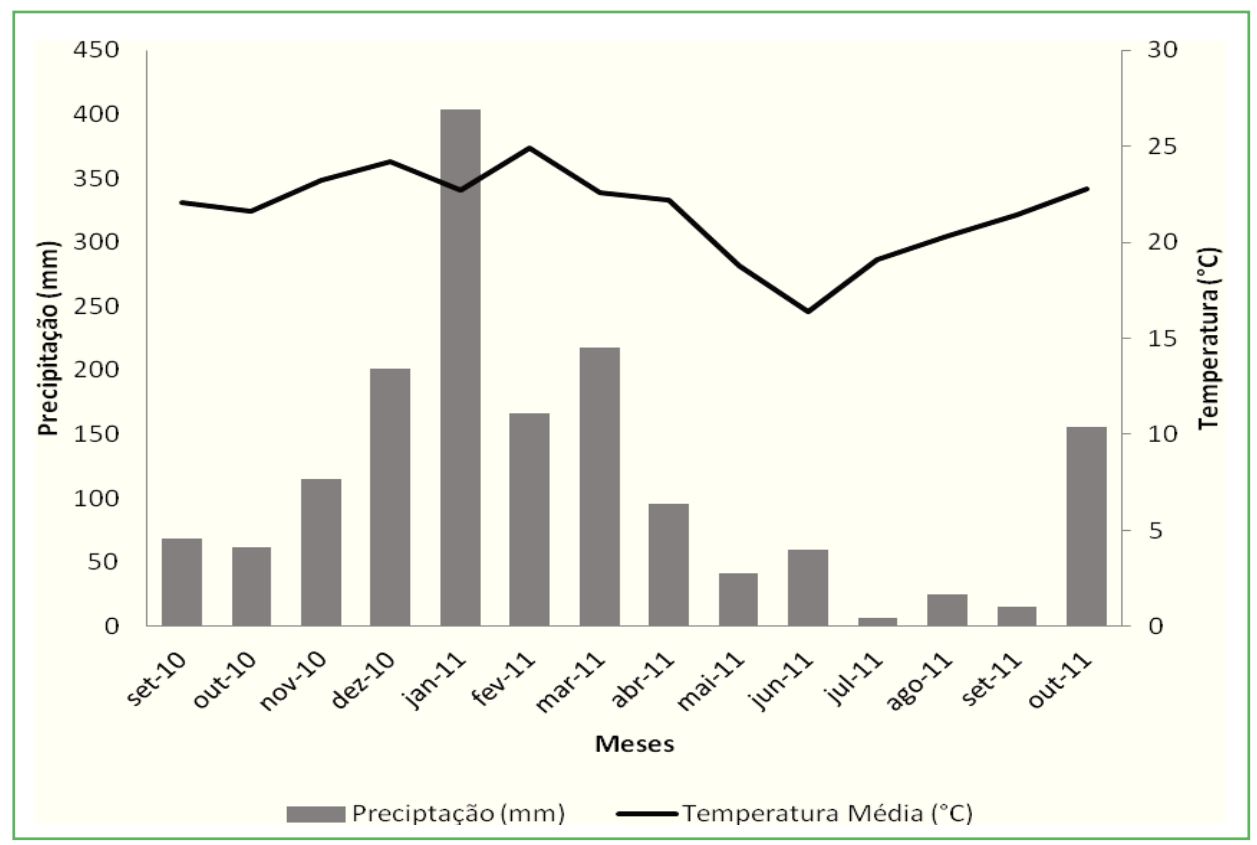

espessura total do limbo. Estimou-se também o diâmetro do pecíolo e da nervura principal e nestes, os raios do floema e xilema, na face abaxial.

O diâmetro e a frequência de elementos de vasos do xilema (vasos $/ \mathrm{mm}^{2}$ ) foram estimados no pecíolo e na nervura principal, nos cortes transversais. O diâmetro do elemento de vaso foi mensurado a partir das suas paredes internas (lúmen), sendo estimado no vaso localizado na região central de cada campo óptico amostrado.

Foram obtidas dez medidas de cada estrutura anatômica, de cada uma das cinco folhas amostradas, totalizando 50 medidas aleatórias por planta, em cada época do ano, utilizando-se o 'software Motic Image Plus'. As comparações foram realizadas entre os períodos chuvoso e seco e, entre os cafeeiros de cada local (coleção e experimento de progênie EP 131), por possuírem idades diferentes. Os dados foram submetidos à análise de variância pelo teste $\mathrm{F}$ e as médias comparadas pelo Tukey, a 5\% de probabilidade.

\section{Resultados}

No presente estudo foi constatada a variação na anatomia foliar entre os períodos sazonais (chuvoso e seco) e entre os cafeeiros, em diversos caracteres anatômicos estudados.

Observou-se (Tabela 1), que após o período de seca, o índice estomático (IE) foi maior na maioria dos cafeeiros, com exceção do Semperflorens e do Geisha, que tiveram diminuição do IE, e do IAC H 8105-7, que não diferiu entre os períodos. No período seco, a densidade de células epidérmicas também diminuiu nos cafeeiros Semperflorens, BA 10, Gláucia e IAC H 8105-7, resultando em células de maiores dimensões $\mathrm{e}$, as dimensões dos estômatos foram menores em Semperflorens, Geisha, 'Catuaí Vermelho IAC 81' (coleção e EP 131) e IAC H 8421-2. A espessura da epiderme na face adaxial das folhas era sempre maior do que na face abaxial (Tabela 2), havendo maior variação entre os cafeeiros na face adaxial. No período seco, a espessura da epiderme, em ambas as faces, foi maior nos cafeeiros Semperflorens e BA 10, enquanto que no Gláucia, as epidermes foram menos espessas.

No período seco, a espessura do parênquima paliçádico aumentou em quase todos os cafeeiros estudados, com exceção do Nacional que diminuiu e, dos cafeeiros Gláucia e IAC H 8421-2 que não variaram (Tabela 2). Os cafeeiros BA 10, Geisha, 
'Catuaí Vermelho IAC 81' (da coleção e do EP 131) e o Semperflorens foram os que apresentaram o parênquima paliçádico mais espesso na seca, sendo maior que $50 \%$ nos Geisha e Catuaí Vermelho IAC 81. A espessura do parênquima esponjoso também aumentou no período seco nos cafeeiros Semperflorens, Nacional, Geisha, IAC H 8105-7 e nas duas plantas da cultivar Catuaí Vermelho IAC 81 (coleção e EP 131), porém, ocorreu uma diminuição na espessura deste parênquima no Gláucia e nenhuma variação no BA 10 (Figura 2).

TABELA 1: Índice estomático (IE), densidade estomática (DE) e densidade das células epidérmicas (CE) e diâmetros, polar e equatorial, dos estômatos de cafeeiros de Coffea arabica L., estimados no período de chuva (abril) e de seca (setembro) do ano de 2011.

\begin{tabular}{|c|c|c|c|c|c|c|c|c|c|c|c|}
\hline \multirow{3}{*}{\multicolumn{2}{|c|}{ Cafeeiros $^{(1)}$}} & \multirow{2}{*}{\multicolumn{2}{|c|}{ IE }} & \multirow{2}{*}{\multicolumn{2}{|c|}{$\begin{array}{c}\text { DE } \\
\text { (estômatos.mm }{ }^{-2} \text { ) }\end{array}$}} & \multirow{2}{*}{\multicolumn{2}{|c|}{$\begin{array}{c}\text { CE } \\
\text { (células epidérmicas. } \\
\text { mm}^{-2} \text { ) }\end{array}$}} & \multicolumn{4}{|c|}{ Diâmetro dos estômatos $(\mu \mathrm{m})$} \\
\hline & & & & & & & & \multicolumn{2}{|c|}{ Polar } & \multicolumn{2}{|c|}{ Equatorial } \\
\hline & & Chuva & Seca & Chuva & Seca & Chuva & Seca & Chuva & Seca & Chuva & Seca \\
\hline \multirow{6}{*}{ Coleção } & 1 & $18,39 \mathrm{abcB}$ & $16,76 \mathrm{aA}$ & $237,22 \mathrm{cB}$ & $76,11 \mathrm{aA}$ & $1061,70 \mathrm{~dB}$ & $872,80 \mathrm{bA}$ & $32,14 \mathrm{bcB}$ & $29,80 \mathrm{bA}$ & $23,77 \mathrm{bB}$ & $19,66 \mathrm{bcA}$ \\
\hline & 2 & $19,16 \mathrm{bcA}$ & $21,83 \mathrm{cB}$ & $210,56 \mathrm{bA}$ & $200,00 \mathrm{bA}$ & $888,30 \mathrm{abB}$ & $718,30 \mathrm{aA}$ & $30,85 \mathrm{bA}$ & $29,53 \mathrm{bA}$ & $22,54 \mathrm{bB}$ & $19,61 \mathrm{bcA}$ \\
\hline & 3 & $18,62 \mathrm{abcA}$ & $22,40 \mathrm{cB}$ & $217,22 \mathrm{bcA}$ & $283,33 \mathrm{cB}$ & $956,10 \mathrm{bcA}$ & $982,80 \mathrm{cA}$ & $29,17 \mathrm{abA}$ & $29,72 \mathrm{bA}$ & $19,86 \mathrm{aA}$ & $19,94 \mathrm{cA}$ \\
\hline & 4 & $18,00 \mathrm{abA}$ & $22,76 \mathrm{cB}$ & $214,44 \mathrm{bcA}$ & $216,67 \mathrm{bA}$ & $982,80 \mathrm{cB}$ & $735,60 \mathrm{aA}$ & $27,75 \mathrm{aA}$ & $27,61 \mathrm{aA}$ & $19,41 \mathrm{aA}$ & $18,50 \mathrm{aA}$ \\
\hline & 5 & $20,09 \mathrm{cB}$ & $18,61 \mathrm{abA}$ & $231,67 \mathrm{bcB}$ & $201,67 \mathrm{bA}$ & $917,80 \mathrm{abcA}$ & $886,70 \mathrm{bA}$ & $28,70 \mathrm{aB}$ & $26,88 \mathrm{aA}$ & $19,61 \mathrm{aB}$ & $18,86 \mathrm{abA}$ \\
\hline & 6 & $17,04 \mathrm{aA}$ & $18,95 \mathrm{bB}$ & $173,89 \mathrm{aA}$ & $208,89 \mathrm{bB}$ & $854,40 \mathrm{aA}$ & $891,70 \mathrm{bA}$ & $28,13 \mathrm{aB}$ & $27,28 \mathrm{aA}$ & $18,64 \mathrm{aA}$ & $18,19 \mathrm{aA}$ \\
\hline \multirow{3}{*}{ EP 131} & 7 & & & & & & & & & 17,89 aA & \\
\hline & 8 & $18,85 \mathrm{aA}$ & $22,05 \mathrm{aB}$ & $181,67 \mathrm{aA}$ & $224,44 \mathrm{bB}$ & $787,22 \mathrm{aA}$ & $800,00 \mathrm{bA}$ & $30,18 \mathrm{bB}$ & $28,17 \mathrm{aA}$ & $20,80 \mathrm{cB}$ & $18,94 \mathrm{aA}$ \\
\hline & 9 & $18,76 \mathrm{aA}$ & $21,15 \mathrm{aB}$ & $202,78 \mathrm{abA}$ & $225,00 \mathrm{bB}$ & $874,40 \mathrm{bA}$ & $846,70 \mathrm{bA}$ & $29,07 \mathrm{bB}$ & $27,80 \mathrm{aA}$ & $19,19 \mathrm{bB}$ & $18,35 \mathrm{aA}$ \\
\hline
\end{tabular}

(1) 1 - Semperflorens (Coleção 6), 2 - BA 10 (Coleção 2), 3 - Nacional (Coleção 9), 4 - Gláucia (Coleção 7), 5 - Geisha (Coleção 1), 6 - 'Catuaí Vermelho IAC 81' (Coleção 4), 7 - IAC H 8105-7 ('Catuaí Vermelho IAC 81' X BA 10) EP 131 planta 91, 8 - IAC H 8421-2 ('Mundo Novo IAC 471-5' X BA 10) EP 131 planta 188, 9 - 'Catuaí Vermelho IAC 81' EP 131 planta 31. Letras minúsculas iguais na vertical entre os cafeeiros de cada local e, maiúsculas iguais na horizontal entre os períodos de chuva e seco, não diferem entre si pelo teste de Tukey a $5 \%$.

TABELA 2: Espessuras $(\mu \mathrm{m})$ das estruturas foliares de cafeeiros de Coffea arabica L., estimadas no período de chuva (abril) e de seca (setembro) do ano de 2011.

\begin{tabular}{|c|c|c|c|c|c|c|c|c|c|c|c|}
\hline \multirow{3}{*}{\multicolumn{2}{|c|}{ Cafeeiros $^{(1)}$}} & \multicolumn{4}{|c|}{ Epiderme } & \multicolumn{4}{|c|}{ Parênquima } & \multirow{2}{*}{\multicolumn{2}{|c|}{ Total do Limbo }} \\
\hline & & \multicolumn{2}{|c|}{ Adaxial } & \multicolumn{2}{|c|}{ Abaxial } & \multicolumn{2}{|c|}{ Paliçádico } & \multicolumn{2}{|c|}{ Esponjoso } & & \\
\hline & & Chuva & Seca & Chuva & Seca & Chuva & Seca & Chuva & Seca & Chuva & Seca \\
\hline \multirow{6}{*}{ Coleção } & 1 & $25,53 \mathrm{abA}$ & $27,68 \mathrm{bB}$ & $20,38 \mathrm{aA}$ & $21,81 \mathrm{abB}$ & $49,35 \mathrm{aA}$ & $65,52 \mathrm{cB}$ & $129,89 \mathrm{aA}$ & $157,77 \mathrm{bcB}$ & $225,14 \mathrm{aA}$ & $272,78 \mathrm{bcB}$ \\
\hline & 2 & $25,76 \mathrm{abcA}$ & $28,21 \mathrm{bB}$ & $22,38 \mathrm{aA}$ & $23,65 \mathrm{bB}$ & $54,85 \mathrm{bA}$ & $72,92 \mathrm{~dB}$ & $135,23 \mathrm{abA}$ & $143,88 \mathrm{bA}$ & $238,22 \mathrm{bA}$ & $268,67 \mathrm{bcB}$ \\
\hline & 3 & 26,86 bcA & $27,58 \mathrm{bA}$ & $21,07 \mathrm{aA}$ & $21,22 \mathrm{aA}$ & $50,81 \mathrm{aB}$ & 45,05 aA & $129,55 \mathrm{aA}$ & $170,46 \mathrm{cB}$ & $228,28 \mathrm{abA}$ & $264,32 \mathrm{bB}$ \\
\hline & 4 & $26,99 \mathrm{bcB}$ & $24,42 \mathrm{aA}$ & $23,06 \mathrm{aB}$ & $21,38 \mathrm{aA}$ & $58,97 \mathrm{cA}$ & $57,01 \mathrm{bA}$ & 141,86 bB & $117,04 \mathrm{aA}$ & $250,88 \mathrm{cB}$ & $219,85 \mathrm{aA}$ \\
\hline & 5 & 25,17 aA & $26,65 \mathrm{bB}$ & $23,21 \mathrm{aA}$ & $21,83 \mathrm{abA}$ & $49,96 \mathrm{aA}$ & $78,08 \mathrm{~dB}$ & $134,86 \mathrm{abA}$ & $158,03 \mathrm{bcB}$ & $233,20 \mathrm{abA}$ & $284,59 \mathrm{cB}$ \\
\hline & 6 & $27,08 \mathrm{cA}$ & $26,91 \mathrm{bA}$ & $22,24 \mathrm{aB}$ & $20,52 \mathrm{aA}$ & $47,88 \mathrm{aA}$ & $76,12 \mathrm{~dB}$ & $134,26 \mathrm{abA}$ & $150,34 \mathrm{bB}$ & $231,47 \mathrm{abA}$ & $273,89 \mathrm{bcB}$ \\
\hline \multirow{3}{*}{ EP 131} & 7 & $24,75 \mathrm{aA}$ & $25,99 \mathrm{bB}$ & $21,38 \mathrm{aA}$ & $22,37 \mathrm{bA}$ & $51,85 \mathrm{bA}$ & $57,27 \mathrm{aB}$ & $129,69 \mathrm{bA}$ & $138,99 \mathrm{aB}$ & $227,67 \mathrm{bA}$ & $244,62 \mathrm{aB}$ \\
\hline & 8 & $27,19 \mathrm{bA}$ & $26,84 \mathrm{bA}$ & $22,79 \mathrm{bB}$ & $21,33 \mathrm{bA}$ & $60,32 \mathrm{cA}$ & 60,03 aA & $134,51 \mathrm{bA}$ & $140,65 \mathrm{aA}$ & $244,81 \mathrm{cA}$ & $248,85 \mathrm{aA}$ \\
\hline & 9 & 24,26 aA & $24,52 \mathrm{aA}$ & $21,17 \mathrm{aB}$ & $19,31 \mathrm{aA}$ & $45,52 \mathrm{aA}$ & $69,24 \mathrm{bB}$ & $121,56 \mathrm{aA}$ & $163,50 \mathrm{bB}$ & $212,51 \mathrm{aA}$ & $276,58 \mathrm{bB}$ \\
\hline
\end{tabular}

(1) 1 - Semperflorens (Coleção 6), 2 - BA 10 (Coleção 2), 3 - Nacional (Coleção 9), 4 - Gláucia (Coleção 7), 5 - Geisha (Coleção 1), 6 - 'Catuaí Vermelho IAC 81' (Coleção 4), 7 - IAC H 8105-7 ('Catuaí Vermelho IAC 81' X BA 10) EP 131 planta 91, 8 - IAC H 8421-2 ('Mundo Novo IAC 471-5’ X BA 10) EP 131 planta 188, 9 - 'Catuaí Vermelho IAC 81' EP 131 planta 31. Letras minúsculas iguais na vertical entre os materiais cafeeiros éticos e, maiúsculas iguais na horizontal entre os períodos de chuva e seco, não diferem entre si pelo teste de Tukey a $5 \%$. 
FIGURA 2: Corte transversal de limbo foliar de cafeeiros de Coffea arabica L., desenvolvidos em períodos de chuva (abril) e de seca (setembro) do ano de 2011.

\section{Chuva}

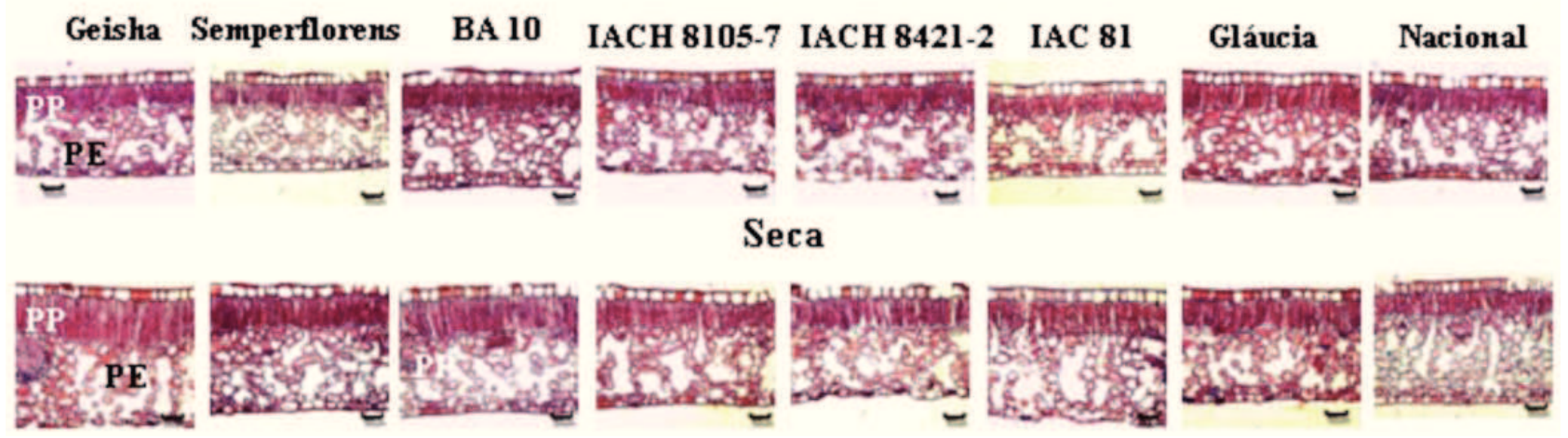

PP = Parênquima paliçádico; PE = Parênquima esponjoso.

Quase todos os cafeeiros apresentaram um limbo mais espesso no período seco, com excessão do Gláucia que diminuiu e do IAC H 8421-2 que não variou a sua espessura. $\mathrm{O}$ aumento da espessura total do limbo dos cafeeiros no período seco foi devido principalmente ao aumento da espessura dos parênquimas paliçádico e esponjoso, exceto no Nacional, que foi devido ao aumento do parênquima esponjoso. No cafeeiro Gláucia a espessura total do limbo foi menor devido a uma diminuição do parênquima esponjoso (Figura 2 e Tabela 2).

O diâmetro do pecíolo aumentou no período seco em quatro cafeeiros (Semperflorens, Geisha, IAC H 8105-7 e IAC H 8421-2) e diminuiu em três (BA 10, Nacional e Gláucia). O diâmetro da nervura principal também aumentou nos cafeeiros Semperflorens, IAC H 8421-2 e 'Catuaí Vermelho 81' (EP 131) e, diminuiu em BA 10 e Nacional (Tabela 3).

No período seco, o raio do floema e do xilema aumentou no pecíolo nos cafeeiros Semperflorens, Geisha, IAC H 8105-7 e IAC H 8421-2, porém, na nervura foliar principal, houve aumento do raio do floema e do xilema nos cafeeiros Semperflorens, Geisha e IAC H 8421-2, enquanto que em Gláucia e 'Catuaí Vermelho IAC 81' do EP 131, aumentou apenas o raio do floema. No pecíolo, os maiores raios do floema no período seco foram observados nos cafeeiros da coleção Semperflorens e Geisha e, no ensaio EP 131, foi no IAC $\mathrm{H}$ 8105-7 e, os maiores raios do xilema foram observados no cafeeiro Geisha e no IAC H 8105-7. Porém, na nervura principal, o maior raio do floema foi observado em 'Catuaí Vermelho IAC 81' (EP 131) e o maior raio do xilema foi observado em Geisha, IAC H 8105-7 e IAC H 8421-2.

A frequência de elementos de vasos do xilema(VF) diminuiu no período seco no cafeeiro Semperflorens, tanto no pecíolo, como na nervura principal, enquanto no cafeeiro Nacional diminuiu somente no pecíolo. $\mathrm{O}$ VF aumentou no período seco no pecíolo dos cafeeiros: Gláucia, 'Catuaí Vermelho IAC 81' (coleção), IAC H 8105-7 e IAC H 8421-2 e, na nervura foliar no Nacional, IAC H 8105-7 e 'Catuaí Vermelho IAC 81' (coleção e EP 131). O cafeeiro Semperflorens apresentou a maior frequência de vasos (VF) no período chuvoso, porém no período seco foi similar aos demais (Tabela 4).

Comparando-se os cafeeiros nos dois períodos, observou-se que no período seco houve um aumento do diâmetro dos vasos (VD) no pecíolo do IAC H 8421-2 e, uma diminuição do VD nos cafeeiros Gláucia e na cultivar Catuaí Vermelho IAC 81 da coleção e do EP 131. Na nervura foliar, o VD aumentou no período seco no Semperflorens e, diminuiu nos cafeeiros BA10, Nacional, Gláucia, 'Catuaí Vermelho IAC 81' da coleção e do EP 131. Os demais cafeeiros analisados não apresentaram diferenças no VD entre os períodos chuvoso e seco (Tabela 4). A variação no VD entre os materiais genéticos foi maior na nervura foliar do que no pecíolo nos dois períodos (chuvoso e seco). 
TABELA 3: Mensurações médias $(\mu \mathrm{m})$ do diâmetro (d) do pecíolo e da nervura foliar principal e das espessuras do floema (rf) e do xilema (rx) da face abaxial de cafeeiros de Coffea arabica L., estimadas após os períodos de chuva (abril) e de seca (setembro) do ano de 2011.

\begin{tabular}{|c|c|c|c|c|c|c|c|}
\hline \multirow{2}{*}{\multicolumn{2}{|c|}{ Cafeeiros $^{(1)}$}} & \multicolumn{2}{|c|}{ d } & \multicolumn{2}{|c|}{$\mathbf{R f}$} & \multicolumn{2}{|c|}{$\mathbf{R x}$} \\
\hline & & Chuva & Seca & Chuva & Seca & Chuva & Seca \\
\hline \multicolumn{8}{|c|}{ PECÍOLO } \\
\hline \multirow{6}{*}{ Coleção } & 1 & $1584,50 \mathrm{aA}$ & $2087,10 \mathrm{bcB}$ & $92,68 \mathrm{aA}$ & $137,05 \mathrm{cB}$ & $132,61 \mathrm{aA}$ & $176,84 \mathrm{bB}$ \\
\hline & 2 & $2280,60 \mathrm{~dB}$ & $1860,00 \mathrm{bA}$ & $130,81 \mathrm{cB}$ & $117,69 \mathrm{bA}$ & $229,77 \mathrm{~dB}$ & $162,08 \mathrm{bA}$ \\
\hline & 3 & $2163,90 \mathrm{cdB}$ & 1977,90 bcA & $131,79 \mathrm{cB}$ & $97,55 \mathrm{aA}$ & $187,34 \mathrm{cB}$ & $141,02 \mathrm{aA}$ \\
\hline & 4 & $1878,40 \mathrm{bB}$ & $1660,50 \mathrm{aA}$ & $97,74 \mathrm{aA}$ & $92,86 \mathrm{aA}$ & $178,46 \mathrm{cB}$ & $164,82 \mathrm{bA}$ \\
\hline & 5 & $1718,00 \mathrm{aA}$ & $1914,70 \mathrm{bB}$ & $110,06 \mathrm{bA}$ & $145,47 \mathrm{cB}$ & $157,19 \mathrm{bA}$ & $225,49 \mathrm{cB}$ \\
\hline & 6 & $2034,00 \mathrm{cA}$ & $1952,60 \mathrm{bcA}$ & $114,58 \mathrm{bA}$ & $115,65 \mathrm{bA}$ & $175,38 \mathrm{cB}$ & $160,13 \mathrm{bA}$ \\
\hline \multirow{3}{*}{ EP 131} & 7 & $1969,20 \mathrm{bA}$ & $2246,20 \mathrm{cB}$ & $116,29 \mathrm{bA}$ & $139,02 \mathrm{cB}$ & $195,73 \mathrm{bA}$ & $222,37 \mathrm{cB}$ \\
\hline & 8 & $1841,50 \mathrm{aA}$ & $1987,90 \mathrm{aB}$ & $102,97 \mathrm{aA}$ & $116,30 \mathrm{aB}$ & $171,98 \mathrm{aA}$ & $181,64 \mathrm{bB}$ \\
\hline & 9 & $2070,30 \mathrm{cA}$ & $2098,20 \mathrm{bA}$ & $120,11 \mathrm{bA}$ & $126,05 \mathrm{bA}$ & $202,88 \mathrm{bB}$ & $160,31 \mathrm{aA}$ \\
\hline \multicolumn{8}{|c|}{ NERVURA FOLIAR } \\
\hline \multirow{6}{*}{ Coleção } & 1 & $789,00 \mathrm{aA}$ & $1081,60 \mathrm{cB}$ & $54,30 \mathrm{bA}$ & $68,06 \mathrm{bB}$ & $85,05 \mathrm{aA}$ & $116,89 \mathrm{abB}$ \\
\hline & 2 & $1126,80 \mathrm{eB}$ & $1030,90 \mathrm{bcA}$ & $69,62 \mathrm{cA}$ & $67,08 \mathrm{bA}$ & $152,50 \mathrm{cB}$ & $126,94 \mathrm{bA}$ \\
\hline & 3 & $1035,20 \mathrm{~dB}$ & $981,60 \mathrm{abA}$ & $64,68 \mathrm{cA}$ & $67,60 \mathrm{bA}$ & $132,82 \mathrm{bB}$ & $108,74 \mathrm{aA}$ \\
\hline & 4 & $914,50 \mathrm{bA}$ & $926,06 \mathrm{aA}$ & $45,50 \mathrm{aA}$ & $50,61 \mathrm{aB}$ & $124,69 \mathrm{bA}$ & $122,24 \mathrm{bA}$ \\
\hline & 5 & $974,10 \mathrm{cA}$ & $1033,20 \mathrm{bcA}$ & 56,09 bA & 63,56 bB & $123,53 \mathrm{bA}$ & $143,07 \mathrm{cB}$ \\
\hline & 6 & $1097,50 \mathrm{eA}$ & $1092,70 \mathrm{cA}$ & $64,46 \mathrm{cA}$ & $67,54 \mathrm{bA}$ & $126,98 \mathrm{bB}$ & $119,55 \mathrm{abA}$ \\
\hline \multirow{3}{*}{ EP 131} & 7 & $1180,50 \mathrm{cA}$ & $1228,60 \mathrm{bA}$ & $73,01 \mathrm{cA}$ & $70,23 \mathrm{aA}$ & $155,16 \mathrm{bA}$ & $162,65 \mathrm{cA}$ \\
\hline & 8 & $916,70 \mathrm{aA}$ & $1126,10 \mathrm{aB}$ & $55,40 \mathrm{aA}$ & $62,44 \mathrm{aB}$ & $135,13 \mathrm{aA}$ & $146,47 \mathrm{bB}$ \\
\hline & 9 & $1072,20 \mathrm{bA}$ & $1157,90 \mathrm{aB}$ & $65,93 \mathrm{bA}$ & $78,82 \mathrm{bB}$ & $137,88 \mathrm{aB}$ & $124,71 \mathrm{aA}$ \\
\hline
\end{tabular}

(1) 1 - Semperflorens (Coleção 6), 2 - BA 10 (Coleção 2), 3 - Nacional (Coleção 9), 4 - Gláucia (Coleção 7), 5 - Geisha (Coleção 1), 6 - 'Catuaí Vermelho IAC 81' (Coleção 4), 7 - IAC H 8105-7 ('Catuaí Vermelho IAC 81' X BA 10) EP 131 planta 91, 8 - IAC H 8421-2 ('Mundo Novo IAC 471-5’ X BA 10) EP 131 planta 188, 9 - 'Catuaí Vermelho IAC 81' EP 131 planta 31. Letras minúsculas iguais na vertical entre os cafeeiros de cada local e, maiúsculas iguais na horizontal entre os períodos de chuva e seco, não diferem entre si pelo teste de Tukey a 5\%.

TABELA 4: Frequências de elementos de vasos do xilema (VF) e diâmetro dos vasos (VD), no pecíolo e na nervura foliar principal, de cafeeiros de Coffea arabica L., estimadas nos períodos de chuva (abril) e de seca (setembro) de 2011.

\begin{tabular}{|c|c|c|c|c|c|c|c|c|c|}
\hline \multirow{3}{*}{\multicolumn{2}{|c|}{ Cafeeiros ${ }^{(1)}$}} & \multicolumn{4}{|c|}{ Pecíolo } & \multicolumn{4}{|c|}{ Nervura Foliar Principal } \\
\hline & & \multicolumn{2}{|c|}{ VF (vasos $\left./ \mathbf{m m}^{2}\right)$} & \multicolumn{2}{|c|}{ VD } & \multicolumn{2}{|c|}{$\mathrm{VF}\left(\operatorname{vasos} / \mathbf{m m}^{2}\right)$} & \multicolumn{2}{|c|}{ VD } \\
\hline & & Chuva & Seca & Chuva & Seca & Chuva & Seca & Chuva & Seca \\
\hline \multirow{6}{*}{ Coleção } & 1 & $2766,30 \mathrm{bB}$ & 1947,10 aA & $16,76 \mathrm{aA}$ & 15,76 aA & $4086,00 \mathrm{bB}$ & $2582,80 \mathrm{abA}$ & $14,51 \mathrm{aA}$ & $18,37 \mathrm{bcB}$ \\
\hline & 2 & $2043,10 \mathrm{aA}$ & $1915,70 \mathrm{aA}$ & $18,29 \mathrm{aA}$ & $18,79 \mathrm{cA}$ & $2284,90 \mathrm{aA}$ & $2314,60 \mathrm{aA}$ & $21,15 \mathrm{cB}$ & $19,53 \mathrm{cA}$ \\
\hline & 3 & $2298,30 \mathrm{aB}$ & $1856,10 \mathrm{aA}$ & $16,91 \mathrm{aA}$ & $16,13 \mathrm{aA}$ & $2439,00 \mathrm{aA}$ & $2985,10 \mathrm{bB}$ & $18,83 \mathrm{bB}$ & $16,54 \mathrm{aA}$ \\
\hline & 4 & $2150,70 \mathrm{aA}$ & $2457,30 \mathrm{cB}$ & $18,05 \mathrm{aB}$ & $16,37 \mathrm{abA}$ & $2521,60 \mathrm{aA}$ & $2661,60 \mathrm{abA}$ & $19,72 \mathrm{bcB}$ & $17,30 \mathrm{abA}$ \\
\hline & 5 & $2124,50 \mathrm{aA}$ & $2052,40 \mathrm{abA}$ & $16,97 \mathrm{aA}$ & $18,22 \mathrm{bcA}$ & $2305,50 \mathrm{aA}$ & $2454,00 \mathrm{aA}$ & $18,71 \mathrm{bA}$ & $19,76 \mathrm{cA}$ \\
\hline & 6 & $2158,90 \mathrm{aA}$ & $2310,20 \mathrm{bcB}$ & $17,85 \mathrm{aB}$ & $16,34 \mathrm{abA}$ & $2363,80 \mathrm{aA}$ & $2716,50 \mathrm{abB}$ & $19,09 \mathrm{bB}$ & $17,62 \mathrm{abA}$ \\
\hline \multirow{3}{*}{ EP 131} & 7 & $1679,60 \mathrm{aA}$ & $1954,80 \mathrm{aB}$ & $19,05 \mathrm{bA}$ & $19,49 \mathrm{bA}$ & $1940,60 \mathrm{aA}$ & $2092,90 \mathrm{aB}$ & $20,70 \mathrm{bA}$ & $20,21 \mathrm{cA}$ \\
\hline & 8 & $1888,20 \mathrm{bA}$ & $2076,10 \mathrm{aB}$ & $17,99 \mathrm{abA}$ & $19,35 \mathrm{bB}$ & $2365,70 \mathrm{cA}$ & $2418,60 \mathrm{bA}$ & $18,65 \mathrm{aA}$ & $18,80 \mathrm{bA}$ \\
\hline & 9 & $1973,30 \mathrm{bA}$ & $2085,50 \mathrm{aA}$ & $17,42 \mathrm{aB}$ & $15,97 \mathrm{aA}$ & $2192,30 \mathrm{bA}$ & $2772,80 \mathrm{cB}$ & $18,61 \mathrm{aB}$ & $17,52 \mathrm{aA}$ \\
\hline
\end{tabular}

(1) 1 - Semperflorens (Coleção 6), 2 - BA 10 (Coleção 2), 3 - Nacional (Coleção 9), 4 - Gláucia (Coleção 7), 5 - Geisha (Coleção 1), 6 - 'Catuaí Vermelho IAC 81' (Coleção 4); 7 - IAC H 8105-7 ('Catuaí Vermelho IAC 81' X BA 10) EP 131 planta 91, 8 - IAC H 8421-2 ('Mundo Novo IAC 471-5’ X BA 10) EP 131 planta 188, 9 - 'Catuaí Vermelho IAC 81' EP 131 planta 31. Letras minúsculas iguais na vertical entre os cafeeiros de cada local e, maiúsculas iguais na horizontal entre os períodos de chuva e seco, não diferem entre si pelo teste de Tukey a $5 \%$. 


\section{Discussão}

Segundo diversos autores (VOLTAN et al., 1992; BOSABALIDIS; KOFIDIS, 2002; NASCIMENTO et al., 2006; MATOS et al., 2009; CÉSAR et al., 2010; LAAJIMI et al., 2011; BALIZA et al., 2012), em ambientes sob condições de estresse hídrico e/ou com maior radiação solar, a frequência de estômatos é maior e suas dimensões menores, contribuindo para o equilíbrio das trocas gasosas (PEARCE et al., 2005). Neste trabalho, os cafeeiros BA 10, Nacional e Gláucia na coleção e o IAC H 8105-7, IAC H 8421-2 e 'Catuaí Vermelho IAC 81', na EP 131, foram os que apresentaram os maiores IE no período seco.

A diminuição do tamanho das células epidérmicas também é uma estratégia adaptativa que contribui para a resistência contra o colapso celular devido à seca e para o controle da transpiração cuticular (BOSABALIDIS; KOFIDIS, 2002), entretanto, tal característica não foi observada nos cafeeiros Semperflorens, BA 10, Gláucia e IAC H 8105-7, que opostamente, apresentaram maiores dimensões no período de seca.

Neste trabalho, quase todos os cafeeiros analisados apresentaram um aumento na espessura do parênquima paliçádico, que é uma resposta adaptativa favorável, por ser um tecido onde ocorre a maior taxa fotossintética, devido à presença de muitos cloroplastos. Outros autores (VOLTAN et al., 1992; NASCIMENTO et al., 2006; GOMES et al., 2008; MATOS et al., 2009; BALIZA et al., 2012), também observaram no cafeeiro arábica, um aumento na espessura do parênquima paliçádico e do esponjoso e, consequentemente do limbo total, em condições de alta radiação solar.

É interessante notar que no cafeeiro IAC H 8421-2 houve um aumento do diâmetro do pecíolo e da nervura principal, porém não ocorreu o mesmo com a espessura total do limbo foliar. Normalmente, o aumento do diâmetro do pecíolo e da nervura principal da folha ocorre devido a um aumento de todos os seus tecidos: parênquima (cortical e medular) e feixes vasculares (xilema e floema). O cafeeiro Semperflorens foi o que apresentou a maior variação entre os períodos chuvoso e seco, tanto no pecíolo, quanto na nervura foliar, indicando ser um cafeeiro com características anatômicas favoráveis para tolerar um estresse hídrico, uma vez que o espessamento dos feixes vasculares possibilita um maior fluxo de seiva elaborada e de água e sais minerais na época seca, conferindo a essas plantas, características adaptativas favoráveis em condições de restrição hídrica. Segundo Carlquist (1977), elementos de vasos mais estreitos resistem mais a altas pressões na coluna d'agua. Portanto, quanto maior a frequência de vasos e menor o seu diâmetro, menor a chance de haver embolia de ar devido ao estresse hídrico, prejudicando seriamente a condução da água e sais minerais na planta.

Tem sido demonstrado por diversos autores, como Bosabalidis e Kofidis (2002), Bacelar et al. (2004; 2006), Pearce et al. (2005), Ennajeh et al. (2010) e Kulkarni et al. (2010), que as espécies ou cultivares desenvolveram diferentes estratégias adaptativas para resistir ao estresse hídrico. Enquanto umas desenvolveram estruturas mais espessas, tais como mesofilo, cutícula adaxial, epidermes ou lâmina foliar, ou um aumento do diâmetro de vasos do xilema; outras apresentaram muitos ou poucos tricomas, área foliar menor ou poucos espaços intercelulares no parênquima esponjoso e maior área de xilema na nervura principal. Estratégia divergente, como menor espessura foliar devido a uma diminuição do parênquima paliçádico e esponjoso, foi observada por Li et al. (1988) e Laajimi et al. (2011), sob condições severas de estresse hídrico. Esta foi acompanhada por um aumento na densidade estomática e no controle da abertura dos estômatos na espécie estudada por Laajimi et al. (2011) e, por uma diminuição na área foliar e no tamanho das células do mesofilo na espécie analisada por Li et al. (1988), uma vez que células menores suportam melhor a pressão de turgor do que as maiores.

A presença ou ausência de características anatômicas adaptativas não resultam obrigatoriamente em tolerância à seca, uma vez que a eficiência dos mecanismos fisiológicos da planta no controle da água possui uma grande importância.

O cafeeiro Geisha, descrito como tolerante por Fazuoli et al. (2012) e como intermediário por Almeida et al. (2007), e o cafeeiro Semperflorens, descrito como tolerante por Almeida et al. (2007), apresentaram diversas características anatômicas de tolerância ao período de baixa disponibilidade hídrica, tais como: 
maiores espessuras do parênquima paliçádico, do limbo total, do raio do floema e do xilema, tanto no pecíolo como na nervura principal. Porém, o diâmetro do vaso do xilema, que era estreito no período chuvoso no cafeeiro Semperflorens, aumentou no período seco, enquanto que no cafeeiro Geisha, permaneceu inalterado. As dimensões dos estômatos diminuíram no período seco nos dois materiais, bem como o índice estomático. Outra característica do cafeeiro Semperflorens era possuir folhas menores do que os outros cafeeiros arábica estudados, característica esta muitas vezes vantajosa em condições de seca.

O cafeeiro BA10 descrito como tolerante à seca por Mazzafera e Carvalho (1987) e como intermediário por Almeida et al. (2007), desenvolveu um parênquima paliçádico e um limbo foliar mais espesso no período seco, menor diâmetro dos vasos do xilema na nervura foliar e o índice estomático aumentou.

A cultivar Catuaí Vermelho IAC 81, foi descrita por Almeida et al. (2007), como sendo de tolerância intermediária à seca. Neste trabalho, as plantas dessa cultivar, analisadas na coleção e no experimento EP 131, apresentaram um aumento no índice estomático e menor diâmetro dos estômatos no período seco. Tiveram também um aumento do parênquima paliçádico e do limbo total neste período porém, o raio do xilema do pecíolo e do limbo foliar diminuiram. A frequência de vasos do xilema aumentou nesse mesmo período, juntamente com uma diminuição do diâmetro dos mesmos.

Os híbridos F1 IAC H 8105-7 e IAC H 84212, originários dos cruzamentos BA10 X 'Catuaí Vermelho IAC 81' e BA10 X 'Mundo Novo IAC 4715, respectivamente, considerados como tolerantes ao estresse hídrico por Almeida et al. (2007), divergiram nas características anatômicas favoráveis à estas condições. Apenas o cafeeiro IAC H 8421-2 apresentou aumento no índice estomático e redução das dimensões dos estômatos, enquanto que em relação à espessura da folha, somente o IAC H 8105-7 aumentou o parênquima paliçádico e o limbo total. Ambos cafeeiros aumentaram o raio dos feixes vasculares no pecíolo, enquanto que na nervura foliar houve aumento dos feixes vasculares apenas no IAC H 8421-2 no período seco. A frequência de vasos do xilema do pecíolo aumentou nos dois híbridos, mas na nervura foliar o aumento ocorreu apenas no híbrido IAC H 8105-7.

O cafeeiro Gláucia, no período seco, apresentou diversos caracteres anatômicos desfavoráveis ao estresse hídrico, tais como diminuição da espessura de ambas epidermes e do mesofilo e, consequentemente, da espessura total do limbo, entretanto, os resultados obtidos nesse trabalho confirmaram as observações preliminares de campo de ser o cafeeiro Nacional sensível à seca.

A partir dos resultados foi possível concluir que os cafeeiros Geisha, Semperflorens, BA 10, IAC H 8105-7 ('Catuaí Vermelho IAC 81' X BA 10), IAC H 8421-2 ('Mundo Novo IAC 471-5' X BA 10) e os da cultivar Catuaí Vermelho IAC 81, foram os que apresentaram características anatômicas favoráveis para tolerar condições de seca, tais como, maiores espessuras do parênquima paliçádico, do limbo total e do raio dos feixes vasculares do pecíolo e na nervura principal, sendo portanto considerados promissores para o programa de melhoramento genético visando tolerância à seca.

\section{Agradecimentos}

Ao Consórcio Brasileiro de Pesquisa e Desenvolvimento do Café (CBP\&D/Café), pelo suporte financeiro para o desenvolvimento desse trabalho.

\section{Referências}

ALMEIDA, J. A. S.; CARVALHO, C. R. L.; SILVAROLLA, M. B.; ARRUDA, F.; BRAGHINI, M. T.; LIMA, V. B.; FAZUOLI, L. C. Caracterização de respostas morfológicas e fisiológicas de diferentes genótipos de Coffea submetidos a estresse hídrico. In: SIMPÓSIO DE PESQUISAS DOS CAFÉS DO BRASIL, 5, 2007, Águas de Lindóia. Resumos... Brasília: EMBRAPA Café; MINASPLAN, 2007. Versão eletrônica.

ARRUDA, F. B.; GRANDE, M. A. Fator de resposta da produção do cafeeiro ao déficit hídrico em Campinas. Bragantia, Campinas, v. 62 , n. 1, p. 139-145, 2003.

BACELAR, E.A.; CORREIA, C. M.; MOUTINHO-PEREIRA, J. M.; GONÇALVES, B. C.; LOPES, J. I.; TORRES-PEREIRA, J. M. G. Sclerophylly and leaf anatomical traits of five field-grown olive cultivars growing under drought conditions. Tree Physiology, Oxford, v. 24, p. 233-239, 2004. 
BACELAR, E. A.; SANTOS, D. L.; MOUTINHO-PEREIRA, J. M.; GONÇALVES, B. C.; FERREIRA, H. F.; CORREIA, C. M. Immediate responses and adaptative strategies of three olive cultivars under contrasting water availability regimes: changes on structure and chemical composition of foliage and oxidative damage. Plant Science, Limerick, v. 170, n. 3, p. 596-605, 2006.

BALIZA, D. P.; CUNHA, R. L.; CASTRO, E. M.; BARBOSA, J. P. R. A. D.; PIRES, M. F.; GOMES, R. A. Trocas gasosas e características estruturais adaptativas de cafeeiros cultivados em diferentes níveis de radiação. Coffee Science, Lavras, v. 7, n. 3, p. 250-258, 2012.

BATISTA, L. A.; GUIMARÃES, R. J.; PEREIRA, F. J.; CARVALHO, G. R.; CASTRO, E. M. Anatomia foliar e potencial hídrico na tolerância de cultivares de café ao estresse hídrico. Revista Ciência Agronômica, Fortaleza, v. 41, n. 3, p. 475-481, 2010

BOSABALIDIS, A. M.; KOFIDIS, G. Comparative effects of drought stress on leaf anatomy of two olive cultivars. Plant Science, Limerick, v. 163, p. 375-379, 2002.

BRODRIBB, T. J.; FEILD, T. S.; JORDAN, G. J. J. Leaf maximum photosynthetic rate and venation are linked by hydraulics. Plant Physiology, Bethesda, v. 144, p. 1890-1898, 2007.

CARLQUIST, S. Ecological factors in wood evolution: a floristic approach. American Journal of Botany, Saint Louis, v. 64, p. 887896, 1977.

CIIAGRO - CENTRO INTEGRADO DE INFORMAÇÕES AGROMETEOROLÓGICAS. CIIAGRO online: resenha agrometeorológica. Disponível em <http:/www.iac.sp.gov.br/New/ ciiagro/>. Acesso em: 19 janeiro 2013.

CÉSAR, F. R. C. F.; MATSUMOTO, S. N.; VIANA, A. E. S.; SANTOS, M. A. F.; BONFIM, J. A. Morfofisiologia foliar de cafeeiro sob diferentes níveis de restrição luminosa. Coffee Science, Lavras, v. 5, n. 3, p. 262-271, 2010.

CUTTER, E. G. Anatomia vegetal: parte I, células e tecidos. 2 ed. São Paulo: Roca, 1986. 304 p

DAMATTA, F. M. Exploring drought tolerance in coffee: a physiological approach with some insights for plant breeding. Brazilian Journal of Plant Physiology, São Paulo, v. 16, p. 1-6, 2004

ENNAJEH, M.; VADEL, A. M.; COCHARD, H.; KHEMIRA, H. Comparative impacts of water stress on the leaf anatomy of a drought-resistant and a drought-sensitive olive cultivar. Journal of Horticultural Science \& Biotechnology, Leuven, v. 85, n. 4, p. 289-294, 2010.

FAZUOLI, L. C.; BRAGHINI, M. T.; SILVAROLLA, M. B.; GUERREIRO FILHO, O. Heterosis and drought tolerance of F1 hybrids between the Catuaí Vermelho cultivar of Coffea arabica and introductions Geisha and Wush-Wush from Ethiopia. In: INTERNATIONAL CONFERENCE ON COFFEE SCIENCE, 24, 2012, San José. Resumos... San José: ASIC, 2012. Versão eletrônica.

GOMES, I. A. C.; CASTRO, E. M. DE; SOARES, A. M.; ALVES J. D.; ALVARENGA, M.I.N.; ALVES,E.; BARBOSA, J. P. R. A. D.; FRIES, D. D. Alterações morfofisiológicas em folhas de Coffea arabica L. cv. "Oeiras" sob influência do sombreamento por Acacia mangium Willd. Ciência Rural, Santa Maria, v. 38, n. 1, p. 109115,2008
GOMES, N. M.; LIMA, L. A.; CUSTÓDIO, A. A. de P. Crescimento vegetativo e produtividade do cafeeiro irrigado no sul do estado de Minas Gerais. Revista Brasileira de Engenharia Agrícola e Ambiental, Campina Grande, v. 11, n. 6, p. 564-570, 2007

GRISI, F. A.; ALVES, J. D.; CASTRO, E. M. DE; OLIVEIRA, C.; BIAGIOTTI, G.; MELO, L. A. DE Avaliações anatômicas foliares em mudas de café 'Catuaí' e 'Siriema' submetidas ao estresse hídrico. Ciências e Agrotecnologia, Lavras, v. 32, n. 6, p. 17301736, 2008 .

JOHANSEN, D. A. Plant microtechnique. New York: McGrawHill, 1940. 523 p.

KULKARNI, M.; SCHNEIDER, B.; RAVEH, E.; TEL-ZUR, N. Leaf anatomical characteristics and physiological responses to short-term drought in Ziziphus mauritiana (Lamk.). Scientia Horticulturae, Amsterdam, v. 124, p. 316-322, 2010.

LAAJIMI, N. O.; BOUSSADIA, O.; SKHIRI, F. H.; SILVA, J. A. T.; REZGUI, S.; HELLALI, R. Anatomical adaptations in vegetative structures of apricot tree (Prunus armenica L.) cv. 'Amor El Euch' grown under water stress. Fruit Vegetable and Cereal Science and Biotechnology, Cambridge, v. 5, n. 2, p. 46-51, 2011.

LI, F. L.; BAO, W. K.; WU, N. Morphological, anatomical and physiological responses of Campylotropis polyantha (Franch.) Schindl. seedlings to progressive water stress. Scientia Horticulture, Amsterdam, v. 127, p. 436-443, 1988

MARCHI, S.; TOGNETTI, R.; MINNOCCI, A.; BORGHI, M.; SEBASTIANI, L. Variation in mesophyll anatomy and photosynthetic capacity during leaf development in a deciduous mesophyte fruit tree (Prunus persica) and an evergreen sclerophyllous Mediterranean shrub (Olea europaea L.). Trees, New York, v. 22, p. 559-571, 2008.

MATOS, F. S.; WOLFGRAMM, R.; GONÇALVES, F. V.; CAVATTE, P. C.; VENTRELlA, M. C.; DAMATTA, F. M. Phenotypic plasticity in response to light in the coffee tree. Environmental and Experimental Botany, Oxford, v. 67, p. 421427, 2009

MAZZAFERA, P.; CARVALHO, A. Produção e tolerância à seca de cafeeiros. Bragantia, Campinas, v. 46, n. 2, p. 403-415, 1987.

MEDIAVILLA, S.; ESCUDEIRO, A.; HEILMEIER, H. Internal leaf anatomy and photosynthetic resource-use efficiency: interspecific and intraespecific comparisons. Tree Physiology, Oxford, v. 21, p. 251-259, 2001.

NASCIMENTO, E. A. DO; OLIVEIRA, L. E. M.; CASTRO, E. M.; DELÚ FILHO, N.; MESQUITA, A. C.; VIEIRA, C. V. Alterações morfofisiológicas em folhas de cafeeiro (Coffea arabica L.) consorciado com seringueira (Hevea brasiliensis Muell. Arg.). Ciência Rural, Santa Maria, v. 36, n. 3, p. 852-857, 2006.

PEARCE, D. W.; MILLARD, S.; BRAY, D. F.; ROOD, S. B. Stomatal characteristics of riparian poplar species in a semi-arid environment. Tree Physiology, Oxford, v. 26, p. 211-218, 2005.

SACK, L.; HOLBROOK, N. M. Leaf hydraulics. The Annual Review of Plant Biology, Palo Alto, v. 57, p. 361-381, 2006.

VOLTAN, R. B. Q.; FAHL, J. I.; CARELLI, M. L. C. Variação na anatomia foliar de cafeeiros submetidos a diferentes intensidades luminosas. Revista Brasileira de Fisiologia Vegetal, Brasília, v. 4, n. 1, p. 99-105, 1992. 